\title{
Soft x-ray spectroscopy study of the element and orbital contributions to the electronic structure of copper hexadecafluoro-phthalocyanine
}

\author{
L. F. J. Piper, S. W. Cho, Y. Zhang, * A. DeMasi, and K. E. Smith ${ }^{\dagger}$ \\ Department of Physics, Boston University, 590 Commonwealth Avenue, Boston, Massachusetts 02215, USA
}

\author{
A. Y. Matsuura \\ European Theoretical Spectroscopy Facility, PCPM, Universite Catholique de Louvain, Bâtiment Boltzmann, Croix du Sud 1, \\ 1348 Louvain-la-Neuve, Belgium \\ C. McGuinness \\ School of Physics, Trinity College Dublin, University of Dublin, Dublin 2, Ireland \\ (Received 26 October 2009; revised manuscript received 7 December 2009; published 6 January 2010)
}

\begin{abstract}
The electronic structure of copper hexadecafluorophthalocyanine $\left(\mathrm{F}_{16} \mathrm{CuPc}\right)$ has been measured using soft $\mathrm{x}$-ray emission spectroscopy and x-ray absorption spectroscopy at the $\mathrm{C}, \mathrm{N}$, and $\mathrm{F} K$ edges. Our element- and orbital-specific measurements confirm that substitution of hydrogen for fluorine leads to profound changes in the electronic environment of the carbon atoms in contrast to that of the nitrogen atoms. These findings are supported by simulated $\mathrm{x}$-ray absorption and emission spectra of $\mathrm{F}_{16} \mathrm{CuPc}$ calculated by density-functional theory methods. The experimental results are directly compared with those of CuPc. Additional information regarding specific carbon sites is obtained from the evolution of the $\mathrm{C} K$-edge resonant x-ray emission spectra of $\mathrm{F}_{16} \mathrm{CuPc}$.
\end{abstract}

DOI: 10.1103/PhysRevB.81.045201

PACS number(s): 71.20.Rv, 78.70.En, 78.70.Dm

\section{INTRODUCTION}

The fundamental properties ${ }^{1}$ and technological applications $^{2}$ of metal phthalocyanines (M-Pc) have been the subject of much research, and indeed M-Pcs are one of the foremost planar organic molecular semiconductors used in many organic optoelectronic devices. ${ }^{3}$ Of this family, there has been increased attention in recent years paid to $\mathrm{CuPc}$ and its derivatives $\mathrm{F}_{16} \mathrm{CuPc}$ and $K$-doped CuPc. ${ }^{4,5}$ While $\mathrm{CuPc}$ semiconductor films are $p$-type in air, ${ }^{6} \mathrm{~F}_{16} \mathrm{CuPc}$ is one of the few organic molecules exhibiting high performance and stability in air under $n$-type operation. ${ }^{7}$ The incorporation of both $n$-type $\mathrm{F}_{16} \mathrm{CuPc}$ and $p$-type $\mathrm{CuPc}$ organic molecules within the active region of a transistor has been shown to be an efficient method for producing ambipolar organic field effective transistors. ${ }^{8}$ Meanwhile, the use of nanometer-scale $\mathrm{F}_{16} \mathrm{CuPc}$ ribbons has been shown to produce high performance air-stable $n$-type transistors, due to the location of the organics' lowest unoccupied molecular orbital (LUMO) compared to the work functions of the $\mathrm{Au}$ and Ag contacts. ${ }^{9}$ Naturally, $\mathrm{CuPc} / \mathrm{F}_{16} \mathrm{CuPc}$ and $\mathrm{F}_{16} \mathrm{CuPc} /$ metal heterojunctions have drawn attention in recent years, e.g., Refs. 10-13 The majority of these studies have employed surface-sensitive photoemission spectroscopy to measure the evolution of the valence-band region and work function following thin-film deposition. Such studies have resulted in emergence of a description of weakly interacting metal-organic and organicorganic interfaces in terms of energetic alignment facilitated by induced interface gap states characterized by a charge neutrality level (CNL). ${ }^{14,15}$ This model has been employed recently to successfully describe both $\left(\mathrm{F}_{4}\right) \mathrm{CuPc} /$ metal and $\mathrm{CuPc} / \mathrm{F}_{4} \mathrm{CuPc}$ interfaces. ${ }^{12}$ The $0.4 \mathrm{eV}$ difference in the CNL of the fluorinated and nonfluorinated molecules was attributed to the change in the transport gap following fluorina- tion, as determined from earlier photoemission and inverse photoemission studies combined with density-functional theory (DFT) calculations of a range of phthalocyanines $\left(\mathrm{H}_{2}-\mathrm{Pc}, \mathrm{CuPc}, \mathrm{F}_{4} \mathrm{Pc}\right.$, and $\left.\mathrm{F}_{16} \mathrm{CuPc}\right)$ by Zahn et al. ${ }^{16}$ These studies provided evidence that the highest occupied molecular orbital (HOMO) and LUMO of fluorinated molecules are extended over the $\mathrm{F}$ atoms, playing an important role in modifying the transport gap, electron affinity and ionization potential ultimately affecting the interfacial properties of the organic molecule. ${ }^{16}$ Clearly further understanding of the role of fluorination is important for future organic-based field effect transistor (OFET) applications, while also providing insight into the role hydrogen plays in the observed proclivity toward $p$-type character of organic semiconductors. ${ }^{17}$ Such a study requires going beyond traditional photoemission techniques.

We report here the results of a soft X-ray absorption spectroscopy (XAS) and soft x-ray emission spectroscopy (XES) study of the element-specific electronic structure of $K$-pristine $\mathrm{F}_{16} \mathrm{CuPc}$ films, and we make direct comparisons with measurements of CuPc films and the results of DFT calculations. XES and XAS are element- and orbitalselective probes of the bulk occupied valence-band states and unoccupied conduction-band states, respectively. We have shown previously that the elemental contributions to the HOMO states of organic molecular thin films can easily be identified using XES. ${ }^{18-22}$ Despite the advantages of XES over traditional PES, only two earlier XES studies of $\mathrm{F}_{16} \mathrm{CuPc}$ and $\mathrm{CuPc}$ exist. ${ }^{4,20}$ While XAS measurements of these two molecules are more common than XES, such studies have typically been employed to investigate the molecular orientation of the molecule at the interface (e.g., Refs. 13 and 23) or have focused on the unoccupied electronic structure of CuPc solely. ${ }^{24,25}$ 
The growth of pristine thin films was confirmed using a combination of XAS and X-ray photoemission spectroscopy (XPS) of the C $1 s$ core level. We observed distinct differences in the electronic structure near the carbon atoms between the two molecules, in agreement with other metal fluorinated and nonfluorinated Pc molecules. ${ }^{10,13,16,26,27}$ While the N $K$-edge XES spectra of the two samples are similar (as are the XAS spectra), we find significant differences between the $\mathrm{C} K$ - $K$-edge XES spectra from the two molecules. This finding is in contrast to earlier XES studies of $\mathrm{F}_{16} \mathrm{CuPc}$ and $\mathrm{CuPc},{ }^{4}$ which we discuss in terms of x-ray induced sample degradation. Our findings are supported by simulations of our experimental XAS and XES spectra based upon DFT calculations obtained using the Stockholm-Berlin (StoBe) implementation of deMon. ${ }^{28}$ Substantive agreement is found between the experimental $\mathrm{C}, \mathrm{N}$, and $\mathrm{F} K$-edge spectra with the simulated XES and XAS spectra for both the fluorinated and the nonfluorinated molecules. The agreement between theory and experiment, in tandem with the spatially, elementally and site-specific resolved nature of the theoretical results, enabled us to assign spectral features in terms of contributions from particular atomic sites, i.e., the site-specific local partial density of states resolved into $\pi\left(\pi^{*}\right)$ or $\sigma\left(\sigma^{*}\right)$ components of the particular molecular orbital. Additional site-specific information was obtained from examining the experimental $\mathrm{C} K$-edge resonant $\mathrm{x}$-ray emission spectroscopy (RXES). In C $K$-edge RXES, specific energetic transitions are favored, especially at threshold, that can provide detailed information regarding the electronic structure at specific carbon sites in organic compounds, as shown previously with benzene, ${ }^{29} \mathrm{CuPc},{ }^{20}$ and aluminum tris-8hydroxyquinoline $\left(\mathrm{Alq}_{3}\right),{ }^{19}$ this arising naturally due to the large site-specific chemical shifts of the carbon $1 s$ binding energy in molecules.

\section{EXPERIMENTAL}

Experiments were performed at the soft x-ray undulator beamline X1B at the National Synchrotron Light Source (NSLS), Brookhaven National Laboratory, which is equipped with a spherical grating monochromator. Core-level XPS spectra were recorded using a Scienta $100 \mathrm{~mm}$ hemispherical electron analyzer, with a $0.25 \mathrm{eV}$ combined energy resolution for the $\mathrm{C} 1 s$ levels. Core-level binding energies were referenced relative to the $\mathrm{Au} 4 f$ photoemission from a $\mathrm{Au}$ foil in electrical contact with the films, and are in agreement with previous $\mathrm{CuPc}$ and fluorinated $\mathrm{CuPc}$ measurements in the literature. ${ }^{10}$ XAS spectra were recorded by the sample drain current technique to obtain the total electron yield (TEY), and were normalized to current from a reference Au coated mesh. The energy scale of the XAS measurements was calibrated using first- and second-order diffraction Ti $L$-edge absorption features of rutile $\mathrm{TiO}_{2} \cdot{ }^{30}$ The energy resolution of the monochromator was set to approximately $0.2 \mathrm{eV}$ for XAS measurements at all edges. XES spectra were recorded with a Nordgren-type grazing incidence grating spectrometer. ${ }^{31}$ The total combined energy resolutions for XES spectra presented here were approximately $0.3 \mathrm{eV}$ near the carbon $K$ edge and were calibrated to emission from $L$ edges of a reference $\mathrm{Ni}$ film in the third-order of diffraction. $^{32}$ The N $K$-edge and F $K$-edge emission spectra were calibrated with the $L_{\alpha} / L_{\beta}$ emission lines of Co (in the second-order of diffraction) and $\mathrm{Fe}$ (in the first-order of diffraction) reference metals. ${ }^{32}$ The energy resolution of the spectrometer was estimated as 0.3 and $0.4 \mathrm{eV}$ for the $\mathrm{N}$ and $\mathrm{F} K$ edges, respectively.

Powders of $\mathrm{F}_{16} \mathrm{CuPc}$ and $\mathrm{CuPc}$ were purchased from Sigma-Aldrich. Thin films were then grown in a custom designed ultrahigh-vacuum organic molecular-beam deposition (OMBD) chamber (base pressure $1 \times 10^{-9}$ torr), attached to the multitechnique soft $\mathrm{x}$-ay spectrometer chamber on beamline X1B. The substrates were $p$-type Si (100) wafers, ultrasonically cleaned in ethanol alcohol before introduction into the OMBD system. The $\mathrm{CuPc}$ and $\mathrm{F}_{16} \mathrm{CuPc}$ were each deposited onto Si substrates from a well outgassed glass crucible, which was resistively heated by a tantalum coil. The substrate temperature was approximately $30{ }^{\circ} \mathrm{C}$ during evaporation. From previous experience with the growth of metal $\mathrm{Pc}$ compounds, ${ }^{20,22}$ the thickness of the deposited films was estimated to be approximately $500 \mathrm{~nm}$. After deposition, the samples were immediately transferred under vacuum into the spectrometer chamber (typical operating pressure of $1 \times 10^{-10}$ torr). High resolution XES measurements require a small photon spot (ca. $40 \mu \mathrm{m})$ with a high photon flux $\left(10^{13} \mathrm{ph} / \mathrm{s}\right)$ on the sample, and long collection times (30-60 $\mathrm{min})$. We have shown previously that these conditions lead to significant beam damage in organic systems, and have successfully addressed this problem by continuously translating the films in front of the beam (at $40 \mu \mathrm{m} / \mathrm{s}$ ) as the spectra are being recorded. ${ }^{20}$ This technique has a dramatic effect on the measured XES spectra from organic thin films, and all (resonant) XES spectra presented in this paper were recorded while continuously translating the samples during data acquisition unless otherwise stated.

\section{CALCULATIONS}

The electronic structure of the $\left(\mathrm{F}_{16}\right) \mathrm{CuPc}$ was calculated by $a b$ initio DFT methods within the StoBe implementation of the deMon package. ${ }^{28}$ It should be noted that unlike most other DFT methods StoBe also has specific implementations for inner-shell spectroscopies and thus can calculate either a XAS spectrum originating from a particular core level in molecules such as phthalocyanines, ${ }^{23}$ or XES spectra at a given core level arising from specific atomic sites within the molecule. The approach is based on self-consistent solutions of the Kohn-Sham (KS) DFT equations using a linear combinations of Gaussian-type orbitals (LCGTO) approach. StoBe was developed in part to describe molecular-orbital changes due to the hydrogen bond formation/breaking in water as measured by XAS and XES. ${ }^{33}$ Our spin-polarized calculations are based on a single geometry optimized $\left(\mathrm{F}_{16}\right) \mathrm{CuPc}$ molecule exhibiting $\mathrm{D}_{4 \mathrm{H}}$ symmetry. In each case the geometry has been optimized using a triple- $\zeta$ valence plus polarization (TZVP) basis set for all C, N (7111/411/1) and a double- $\zeta$ valence plus polarization (DZVP) basis (63321/531/311) for $\mathrm{Cu}$ and $\mathrm{H}$ (41). Auxiliary basis sets were applied to fit the electron density and the exchange- 
correlation potential. All the computations were performed for a single $\left(\mathrm{F}_{16}\right) \mathrm{CuPc}$ molecule, and included the generalized gradient approximation correction of Perdew and Wang ${ }^{34}$ and the exchange correlational of Becke ${ }^{35}$ throughout the spin-polarized calculations. We note that for M-Pcs, the molecule-molecule interaction is generally weak in the organic crystalline solid and therefore the measured emission spectra may be compared to that calculated from an isolated single molecule. The simulated $\mathrm{x}$-ray emission spectra are calculated using the ground-state molecular orbitals, in preference to transition state or core-hole orbitals, as has been noted previously for x-ray emission. ${ }^{36}$ The calculated dipole allowed transitions between the $1 s$ core hole on the respective unique chemical sites of the $\mathrm{C}, \mathrm{N}$, and $\mathrm{F}$ atoms and their respective site-specific projections of the occupied $2 p$ atomic orbitals, which form part of the occupied molecular orbitals or occupied partial density of states, result in a spectrum of transitions for each atomic site. The calculated transition energies reflect the KSorbital energies of both the core hole and the occupied molecular orbitals and thus a rigid shift of the calculated transitions can be used to correct for the difference between the KS orbital derived transition energies and the real transition energies. A proper calculation of the corelevel binding energies is through a $\Delta \mathrm{KS}$ approach, where the total energy of the molecule with a specific core hole is differenced from that of a molecule with fully occupied core orbitals, ${ }^{37}$ and these ionization energies been used here to correct the calculated XES and XAS spectra. For each elemental $K$ edge, $\mathrm{C}, \mathrm{N}$, and $\mathrm{F}$, the combined XES spectra presented here are thus the sum of the equally weighted contributions from the four differing $\mathrm{C}$ sites, the two $\mathrm{N}$ sites, and the two F sites, respectively. As the calculations are for a single molecule it is instructive to consider the angular dependence of the simulated XES with respect to the plane of the molecule as will be discussed later. Although not shown here the calculated occupied partial density of states are in good agreement with the results from other calculations. . $2,38^{2}$

In addition to the XES spectra, XAS spectra have also been calculated using the StoBe package. For the XAS calculations the spectra were obtained in a transition state potential with a series of calculations for each unique molecular $\mathrm{C}, \mathrm{N}$, or $\mathrm{F}$ site in each spin channel. The $1 s$ electrons of each core excited $\mathrm{C}, \mathrm{N}$, or $\mathrm{F}$ atom are described by the IGLO-iii triple- $\zeta$ orbital basis of Kutzelnigg et al. ${ }^{39,40}$ In each case, the $1 s$ electrons of all other elements are incorporated into an effective core-potential appropriate to the remaining non- $1 s$ electrons. The resulting core-unoccupied transitions for all sites and for both spin channels are calculated as XAS spectra. In these the polarization vector is either perpendicular to the plane of the molecule ( $p$-polarized) and hence resonant with $\pi^{*}$ states, or in the plane of the molecule ( $s$-polarized) and resonant with $\sigma^{*}$ states. For the latter, the response to the linearly polarized light is averaged over all directions within the plane of the molecule. The transition energies of the XAS spectra, calculated in the transition state potential, are further corrected by similar $\Delta \mathrm{KS}$ calculations to compute the relaxed ionization energy where in addition the first transition to an unoccupied molecular orbital was adjusted to equal the difference in total energy between the ground state and the core excitation to the lowest unoccupied molecular orbital or

\section{(a) $\mathrm{F}_{16} \mathrm{CuPc}$}

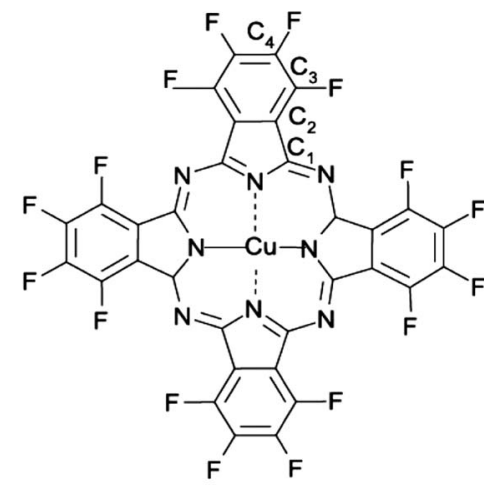

(b) $\mathrm{CuPc}$

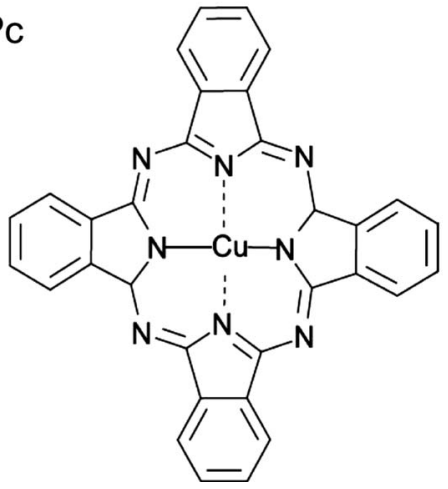

FIG. 1. Schematic representation of the (a) $\mathrm{F}_{16} \mathrm{CuPc}$ and (b) $\mathrm{CuPc}$ molecules. The four distinct carbon environments are labeled $\mathrm{C}_{1}-\mathrm{C}_{4}$ for $\mathrm{F}_{16} \mathrm{CuPc}$. The distinct $\mathrm{N}$ and $\mathrm{F}$ sites are also labeled.

LUMO with the presence of an excited electron in the other spin channel. The XAS spectra presented here are in each case then the equally weighted sum over the differing chemical sites within the molecule. The XAS transitions are Gaussian broadened by $0.5 \mathrm{eV}$ full width at half maximum (FWHM) approximating both core-hole lifetime and instrumental resolution below the ionization threshold, from where the broadening increases to $4.5 \mathrm{eV}$ over a $30 \mathrm{eV}$ range above the ionization potential, similar to the method employed by Kolczewski et al. ${ }^{37}$ An exception to this is the $\mathrm{F} K$-edge $\mathrm{XAS}$, as will be discussed below. This approach to $\mathrm{F}_{16} \mathrm{CuPc}$ has already been employed to model the observed $\mathrm{N} K$-edge XAS of monolayer thin films of $\mathrm{CuPc},{ }^{23}$ where it has obtained good agreement.

\section{X-RAY PHOTOEMISSION}

Figure 1 displays schematics of the molecular structure of $\mathrm{CuPc}$ and $\mathrm{F}_{16} \mathrm{CuPc}$. The primary difference between the two molecules is the substitution of hydrogen for fluorine of the benzene ring carbon atoms, with no significant changes in bond lengths. The atoms are numbered $\mathrm{C}_{1}-\mathrm{C}_{4}$, with the atoms $\mathrm{C}_{1,2}$ equivalent for the two molecules and $\mathrm{C}_{3,4}$ bonded to fluorine (hydrogen) for $\mathrm{F}_{16} \mathrm{CuPc}(\mathrm{CuPc})$ Additional features in the photoemission spectra of the $\mathrm{C} 1 s$ core level due to the substitution for highly electronegative fluorine are expected, with little effect on the $\mathrm{Cu}$ and $\mathrm{N}$ environments.

Figure 2 presents our $\mathrm{C} 1 s$ photoemission spectra of our $\mathrm{F}_{16} \mathrm{CuPc}$ films, along with that from a reference $\mathrm{CuPc}$ film. 


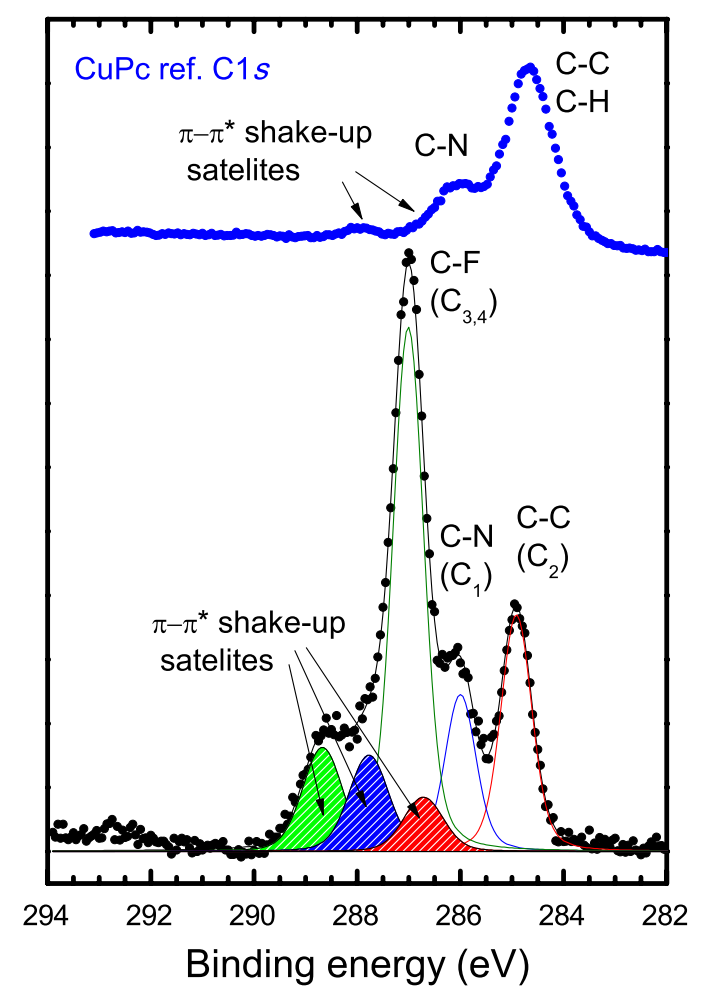

FIG. 2. (Color online) The $\mathrm{C} 1 s$ XPS spectrum of $\mathrm{F}_{16} \mathrm{CuPc}$ (filled circles). The Voigt function fit and its components are also displayed, identifying the three carbon environments associated with the $\mathrm{C}-\mathrm{N}\left(\mathrm{C}_{1}\right), \mathrm{C}-\mathrm{C}\left(\mathrm{C}_{2}\right)$, and $\mathrm{C}-\mathrm{F}\left(\mathrm{C}_{3}, \mathrm{C}_{4}\right)$, bonds and their respective $\pi-\pi^{*}$ shake-up satellites. For reference, the C $1 s$ XPS spectrum of $\mathrm{CuPc}$ is shown above.

Three core-level states are clearly observed for $\mathrm{F}_{16} \mathrm{CuPc}$, along with three accompanying shake-up satellites associated with kinetic-energy loss of photoelectrons due to simultaneously excited $\pi-\pi^{*}$ excitations, i.e., HOMO to LUMO. The common method of fitting the carbon $1 s$ XPS of nonfluorinated $\mathrm{M}-\mathrm{Pc}$, typically considers only two core-level states, i.e., pyrrole $\left(\mathrm{C}_{1}\right)$ and benzene $\left(\mathrm{C}_{2,3,4}\right)$ related peaks along with their corresponding shake-up satellites. ${ }^{41-44}$ However, it has recently been reported that improved curve fitting can be made by splitting the benzene carbon peak into two weighted components (along with their corresponding shake-up satellites) $\sim 0.4 \mathrm{eV}$ apart from each other. ${ }^{45}$ This separation could be either due to the electronegative difference between the local environments of the $\mathrm{C}_{2}$ and $\mathrm{C}_{3,4}$ carbon atoms of the $\mathrm{CuPc}$ (i.e., three compared to two $\mathrm{C}$ nearest neighbors) giving an additional chemical shift, ${ }^{46,47}$ due to the excitation of an in-plane $\mathrm{C}-\mathrm{H}$ stretching vibrational mode following the excitation of the photoelectron from the $\mathrm{C} 1 \mathrm{~s}$ level for the nonfluorinated $\mathrm{Pc},{ }^{25}$ or due to differences in screening on each of the $\mathrm{C}$ atoms. In the fluorinated case the in-plane $\mathrm{C}-\mathrm{F}$ stretching mode is reduced to $\sim 0.18 \mathrm{eV}$ from $0.38 \mathrm{eV}$ for $\mathrm{C}-\mathrm{H}$ in $\mathrm{CuPc}$ and an additional peak due to this vibrational loss is no longer vital to achieve a good fit to the $\mathrm{C} 1 \mathrm{~s}$ photoemission spectrum.

For the $\mathrm{F}_{16} \mathrm{CuPc}$ molecule, the energetic splitting of $\sim 2 \mathrm{eV}$ between the $\mathrm{C}_{2}$ and $\mathrm{C}_{3,4}$ carbons is certainly due to the highly electronegative fluorine atoms giving rise to a chemical shift. There is also a slight shift toward higher binding energies of the $\mathrm{C}-\mathrm{C}$ (i.e., $\mathrm{C}_{2}$ ) and pyrrole $\mathrm{C}-\mathrm{N}$ (i.e., $\mathrm{C}_{1}$ ) related peaks in comparison with the CuPc molecule. We note agreement with our measurements and previous studies of the $\mathrm{C} 1 \mathrm{~s}$ of $\mathrm{F}_{16} \mathrm{CuPc}$ by Ottaviano et al. ${ }^{48}$ and Peisert et al. ${ }^{10}$ both in terms of spectral shape and binding energies. and We also observe a shift of $0.4 \mathrm{eV}$ toward higher binding energies between the fluorinated and nonfluorinated molecules, which may be associated with their respective $n$-type and $p$-type characters, consistent with the recent work of Chen et al. regarding the shift between the $\mathrm{C}-\mathrm{C}$ species of the two molecules. ${ }^{13}$ For the additional analysis of the $\mathrm{C} 1 \mathrm{~s}$ core-level line shapes we used Voigt functions to fit the $\mathrm{F}_{16} \mathrm{CuPc} \mathrm{C} 1 s$ XPS spectrum with a Shirley-type background subtracted. The fitting procedure assumed the same Lorentzian and Gaussian parameters for each the main peak components, using similar parameters as reported by Peisert et al. ${ }^{10}$ (i.e., a FWHM of $0.20 \mathrm{eV}$ and $0.6 \mathrm{eV}$ for the Lorentzian and Gaussian parts, respectively). The fitted intensity ratios for the different carbon features agree well with the expected $\sim 2: 2: 4$ (C-C, C-N, C-F) ratio for this molecule. The shake-up satellite features were each described by Gaussian peaks at a distance of 1.7-1.75 eV from the corresponding main line for $\mathrm{F}_{16} \mathrm{CuPc}$, in agreement with previous studies by Peisert et al. ${ }^{10}$ This approach was favored over that employed by Ottaviano et al., where the shake-up satellites from the $\mathrm{C}-\mathrm{N}$ and $\mathrm{C}-\mathrm{F}$ components only were considered. ${ }^{48}$ This energetic separation of the shake-up satellites corresponds well to the transport gap of $1.8 \mathrm{eV}$ for $\mathrm{F}_{16} \mathrm{CuPc},{ }^{16}$ confirming its $\pi-\pi^{*}$ origin. We also find that the largest relative intensity of the $\pi-\pi^{*}$ shake-up satellite feature compared to the main line, is associated with the $\mathrm{C}-\mathrm{N}$ species. This finding was considered by Peisert et al. to be associated with the large contribution of the $\mathrm{C}-\mathrm{N}$ species for both the HOMO and LUMO. ${ }^{10}$ We shall show that this explanation is further supported by our experimental N $K$-edge XES and XAS, where the HOMO and LUMO states are clearly identified and the $\mathrm{N}$ states must contribute strongly to both of these features.

\section{X-RAY ABSORPTION}

Figure 3 displays the XAS of the $\mathrm{C}$ and $\mathrm{N} K$ edges of the two molecules. A large difference is noted between the fluorinated and nonfluorinated molecule in the $\mathrm{C} K$-edge spectra. This is in contrast to the $\mathrm{N} K$-edge spectra, which are quite similar for the two molecules. We also note that all of our absorption spectra are in agreement with recent XAS measurements of $\mathrm{CuPc}$ and $\mathrm{F}_{16} \mathrm{CuPc}^{13,24,27}$ We attribute the energetic shift of peaks (a) and (b) in $\mathrm{F}_{16} \mathrm{CuPc}$ compared to their counterparts of the $\operatorname{CuPc}\left[\left(\mathrm{a}^{\prime}\right)\right.$ and $\left.\left(\mathrm{b}^{\prime}\right)\right]$ as due to a combination of the shift in the $\mathrm{C} 1 s$ binding energies of the $\mathrm{C}-\mathrm{N}$ and $\mathrm{C}-\mathrm{C}$ bonds in the XPS due to their respective doping (as discussed previously) and change in the transport gap upon fluorination. ${ }^{16}$ Plotting the $\mathrm{C} K$-edge XAS of both molecules on a common binding-energy axis (i.e., rigidly subtracting the core-level binding energy of the C-C peak) results in the transition to the LUMO lying energetically below the Fermi level. This can be explained in terms of a Coulomb interaction between the excited electron and the $1 s$ core hole 
(a)

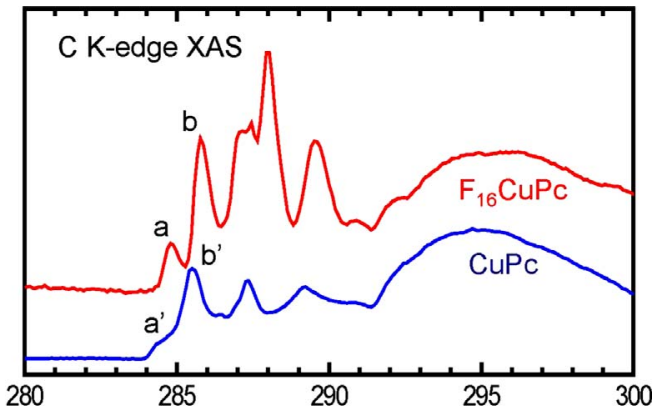

(b)

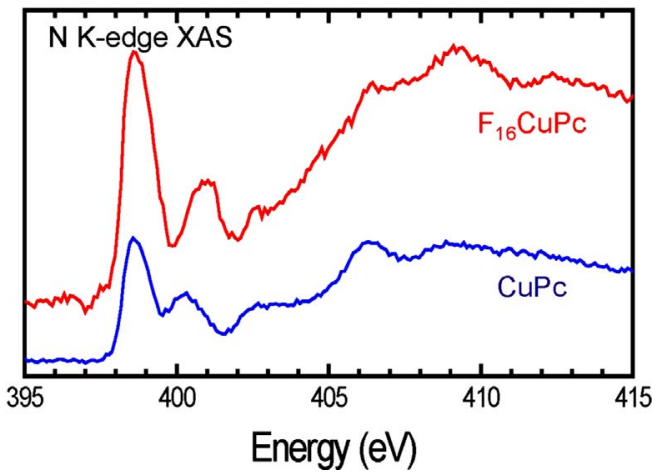

FIG. 3. (Color online) The (a) $\mathrm{C}$ and (b) $\mathrm{N} K$-edge x-ray absorption spectra of $\mathrm{F}_{16} \mathrm{CuPc}$ (red lines). Also displayed underneath each spectrum is the CuPc XAS for the $\mathrm{C}$ and $\mathrm{N} K$ edges (blue lines).

created during $\mathrm{x}$-ray absorption process, and has been observed for other phthalocyanines and other organics, e.g., $\mathrm{H}_{2} \mathrm{Pc}$ (Ref. 49) and $\mathrm{Alq}_{3} \cdot{ }^{19}$

Figure 4(a) displays the angular dependence of the C $K$ edge of the $\mathrm{F}_{16} \mathrm{CuPc}$ molecule in order to determine the azimuthally averaged tilt of the molecules with respect to the substrate. The C $K$-edge has been favored over the $\mathrm{N} K$-edge, since the latter has a nonzero $\sigma^{*}$ contribution in the lowest energy peaks of the $\mathrm{N} K$-edge XAS spectrum. This can result in an overestimation of the tilt, as recently reported from $\mathrm{N} \mathrm{K}$-edge XAS of thin well-ordered $\mathrm{CuPc}$ films in combination with StoBe simulations of the absorption spectra. ${ }^{23}$ Figure 4(b) displays the variation of the relative intensity of the $\pi^{*}: \sigma^{*}$ peaks in Fig. 4(a). The intensity (I) of the $\pi^{*}$ resonance in the XAS spectra is related to the tilt angle $\alpha$ of the $\mathrm{F}_{16} \mathrm{CuPc}$ molecular plane with respect to the substrate plane and the photon incidence angle $\theta$ by $^{50}$

$$
I(\theta) \propto 1+\frac{1}{2}\left(3 \cos ^{2} \theta-1\right)\left(3 \cos ^{2} \alpha-1\right) .
$$

From comparison with the calculated variation for a range of tilt angles, we conclude an azimuthally averaged tilt of $45^{\circ}$ with respect to the substrate, as shown in Fig. 4(b).

For large organic compounds such as the phthalocyanines, the molecular orbitals are strongly distorted upon the core excitation involved in the XAS process, as shown for $\mathrm{H}_{2}-\mathrm{Pc} .{ }^{49}$ The large site dependence of the electron-hole interaction means that the $\mathrm{C} K$-edge XAS does not directly represent the ground-state density of unoccupied states and interpretation of the $\mathrm{C} K$-edge XAS in this manner can be misleading. This is especially true to the $\mathrm{F}_{16} \mathrm{CuPc}$ case. In the

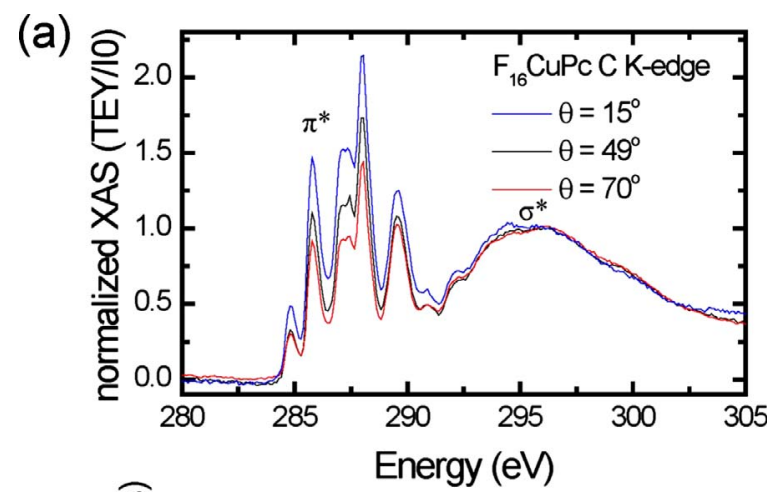

(b)

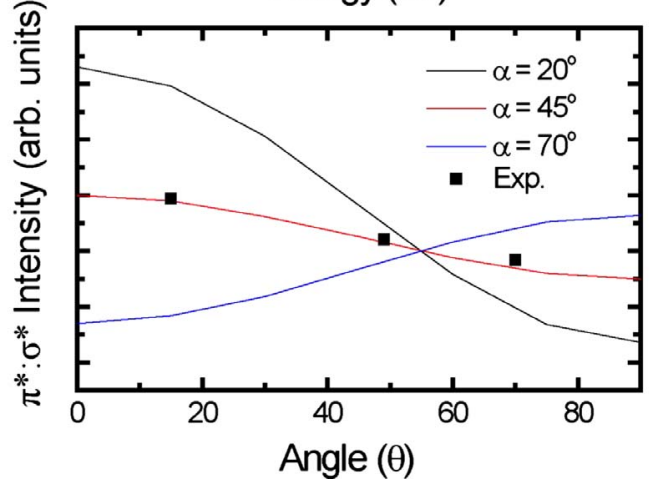

FIG. 4. (Color online) (a) Angular variation in the C $K$-edge XAS of F16CuPc. (b) The plotted variation of the experimental $\pi^{*}: \sigma^{*}$ peak intensity (black square) against the calculated variation for a range of planar tilt angles.

$\mathrm{CuPc}$ case the coincidence of the core-level shifted $\mathrm{C} K$-, $\mathrm{N} K$-, and $\mathrm{Cu}_{L_{3}}$-edge XASs has been interpreted using ground-state DFT calculations of the unoccupied states, where it was concluded that the same unoccupied $\pi^{*}$ states (localized on the pyrrole carbons) are available for transitions from both the $\mathrm{C} 1 s$ and $\mathrm{N} 1 s$ core levels in $\mathrm{CuPc}^{24,38,51}$ Although the assignment of individual atom contributions to the $\mathrm{C} K$-edge XAS were only determined following XAS simulations within $a b$ initio static-exchange approach calculations. ${ }^{25}$ Likewise, it is necessary simulate the absorption spectra of $\mathrm{F}_{16} \mathrm{CuPc}$ in order to fully consider the initial and final states for assigning specific atomic contributions necessary for interpreting the $\mathrm{C} K$-edge RXES spectra discussed later.

Figure 5 compares the $\mathrm{C}, \mathrm{N}$, and $\mathrm{F} K$-edge XAS of $\mathrm{F}_{16} \mathrm{CuPc}$ with the corresponding XAS simulations. An incidence angle of $45^{\circ}$ is presented in each case, corresponding to roughly equal $\pi^{*}$ and $\sigma^{*}$ contributions as was shown in Fig. 4(b). The simulated XAS spectra for $\mathrm{F}_{16} \mathrm{CuPc}$ are plotted underneath each experimental spectrum, reflecting the separate simulated $\pi^{*}$ and $\sigma^{*}$ contributions perpendicular to the plane of the molecule and in the plane of the molecule respectively. Overall very good agreement is seen between the experimental and simulated absorption spectra for all three edges (including the $\mathrm{F} K$ edge). For the $\mathrm{C} K$-edge XAS in Fig. 5(a), the very first feature (a) is a transition to the $e_{\mathrm{g}}$ LUMO of the phthalocyanine originating from the $\mathrm{C}-\mathrm{C}$ "link" phenyl site $\left(\mathrm{C}_{2}\right)$. The lowest transition energy from the pyrrole site $\left(\mathrm{C}_{1}\right)$ occurs $1.0 \mathrm{eV}$ higher, i.e., feature (b), similar to the $1.2 \mathrm{eV}$ chemical shift in the $1 s$ binding ener- 

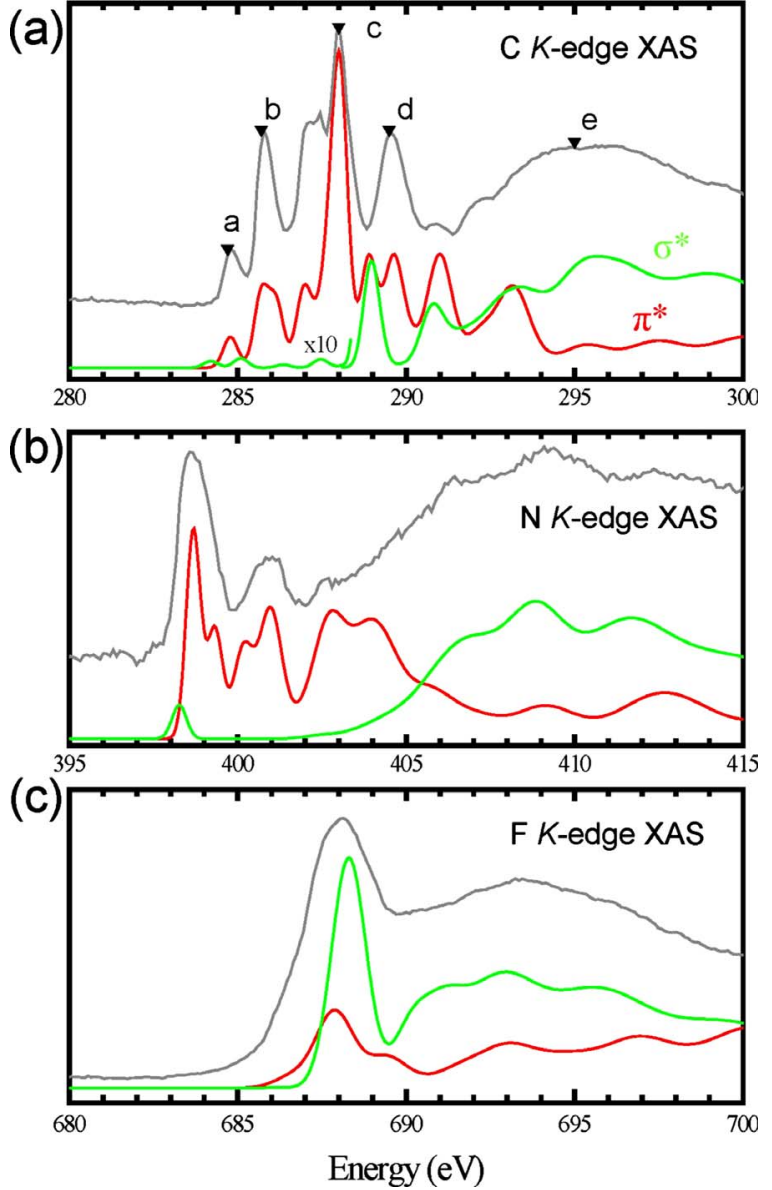

FIG. 5. (Color online) The experimental and simulated x-ray absorption spectra for the (a) C $K$ edge, (b) N $K$ edge, and (c) F $K$ edge of $\mathrm{F}_{16} \mathrm{CuPc}$. The $\pi^{*}$ (red) and $\sigma^{*}$ (green) contributions to the total (black) simulated spectra are shown for each $K$ edge. The resonant excitation energies used for the $\mathrm{C} K$ edge RXES are also shown for the $\mathrm{C} K$-edge as triangles marked (a)-(e).

gies between this and the phenyl sites. For $\mathrm{CuPc}$, the $0.2 \mathrm{eV}$ difference has been associated with excitonic effects resulting from the greater charge localization of the states on the pyrrole carbons compared to the benzene carbons. ${ }^{38}$ Transitions to the LUMO and LUMO+1 involving the outer carbon sites $\left(\mathrm{C}_{3,4}\right)$ contribute the greatest at higher energies i.e., the shoulder and peak of feature (c), respectively. This assignment is especially important for interpreting the C $K$-edge RXES spectra.

The N $K$-edge XAS is broadly similar to that obtained for $\mathrm{CuPc}$ (not shown here) and as is evident from comparison with the XAS work of Chen et al. ${ }^{13}$ of monolayers of $\mathrm{F}_{16} \mathrm{CuPc}$, the experimental spectrum in Fig. 5(b) has approximately equal contributions of $s$-polarized and $p$-polarized absorption. As in the case of the N $K$-edge XAS of CuPc (Ref. 23) we calculated an in-plane $\sigma^{*}$ component of the XAS in the energy region of the $\pi^{*}$ resonances. This arises from transitions on the inner nitrogen to those in-plane $2 p$ orbitals bonding with the unoccupied $\mathrm{Cu} 3 d_{x^{2}-y^{2}}$ orbital. The transition energy of this in-plane $\sigma^{*}$ peak is calculated to be lower than the first $\pi^{*}$ resonance which is derived from the aza mesobridging nitrogens, but again this is not the case in prac- tice, as it occurs higher. This discrepancy indicates that a greater on-site Coulomb energy for the $3 d$ orbitals should be included. For both $\mathrm{F}_{16} \mathrm{CuPc}$ and $\mathrm{CuPc}$, the shift from the calculated position of this $\sigma^{*}$ peak to the position observed by Chen et al. is the same, whereas the peak of the $\pi^{*}$ resonance is observed to occur at a higher transition in $\mathrm{F}_{16} \mathrm{CuPc}$ by $\sim 0.5 \mathrm{eV}$. The simulated XAS spectra are shown for comparison in Fig. 5(b) where we note a coincidental positioning of a fluctuation in the pre-edge TEY at $\sim 397.5 \mathrm{eV}$ with the calculated $\sigma^{*}$ XAS spectrum. The observed $\sigma^{*}$ transition should occur $\sim 0.45 \mathrm{eV}$ higher in excitation energy than the calculation depicts. It may be that the shape of the $\mathrm{N} K$-edge $\pi^{*}$ transition reflects vibronic coupling as was the case for the $\mathrm{N} \mathrm{K}$ edge of $\mathrm{H}_{2} \mathrm{Pc}$ which upon analysis reflected a vibrational envelope of transitions including $\mathrm{C}-\mathrm{N}$ and $\mathrm{C}-\mathrm{C}$ stretching modes. ${ }^{49}$

The measured F $K$-edge XAS, together with the simulated $\sigma^{*}$ and $\pi^{*}$ XAS spectra are shown in Fig. 5(c). As distinct from the earlier simulated spectra at the $\mathrm{C}$ and $\mathrm{N} K$-edges, the calculated transitions at the $\mathrm{F} K$-edge are broadened considerably more than the $0.5 \mathrm{eV}$ noted earlier, and are instead broadened by a Gaussian of $1.2 \mathrm{eV}$ FWHM, increasing steadily to $6.5 \mathrm{eV}$ above the ionization potential. This broadening is to account for the vibrational envelope associated with excitation on any of the $\mathrm{F}$ sites as they have significantly more freedom in this respect than any of the more constrained carbon or nitrogen atoms within the molecules. There is a significant variation of $\mathrm{F} 1 s$ core-level linewidths associated with either simple molecules ${ }^{52}$ or fluorinated nanotubes ${ }^{53}(1.2-2.6 \mathrm{eV})$, and with the value used here at the lower end of this scale a very satisfactory agreement between theory and experiment is reached.

\section{X-RAY EMISSION}

XES records the $\mathrm{x}$-ray fluorescence resulting from the transition of an electron from the valence band into an empty core-hole state created by the excitation of an electron out of the system (i.e., above threshold) ${ }^{54,55}$ Element selectivity and dipole selection rules $(\Delta \mathrm{l}= \pm 1)$, ensures that XES yields information regarding transitions from the core-hole to the occupied element-specific partial density of states (PDOS) for the $\mathrm{C}, \mathrm{N}$ and $\mathrm{F} K$-edges (thus justifying the computational approach employed). For organic compounds, soft $\mathrm{x}$-ray emission spectra can be used to determine the local $p$-orbital character of HOMO states of the molecule. This is best reflected by the $\mathrm{N}$ and $\mathrm{F} K$-edge nonresonant $\mathrm{x}$-ray emission spectra of $\mathrm{F}_{16} \mathrm{CuPc}$ in Fig. 6. In these cases, the distribution of core-level binding energies between the dissimilar chemical sites is relatively small and consequently one can directly compare the respective calculated transitions from the $p$-like orbital distribution to the measured spectra. In Fig. 6(a), the general spectral shape of the $\mathrm{N} K$-edge of $\mathrm{F}_{16} \mathrm{CuPc}$ [i.e., peaks (i)-(v)] is in agreement with $\mathrm{N} K$ edge of $\mathrm{CuPc}$ films, ${ }^{20}$ as expected due to lack of influence the fluorine has on the $\mathrm{N}$ environment of $\mathrm{F}_{16} \mathrm{CuPc}$. From direct comparison with earlier $\mathrm{N} K$-edge emission spectra of $\mathrm{F}_{16} \mathrm{CuPc}$ by Kurmaev et al. ${ }^{4}$ we observe a far stronger dominant peak (i) with features (iii)-(v) more prominent in our 
(a)

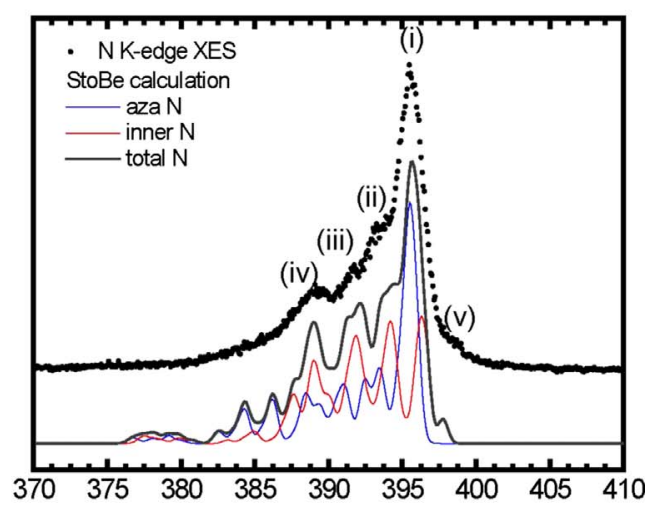

(b)

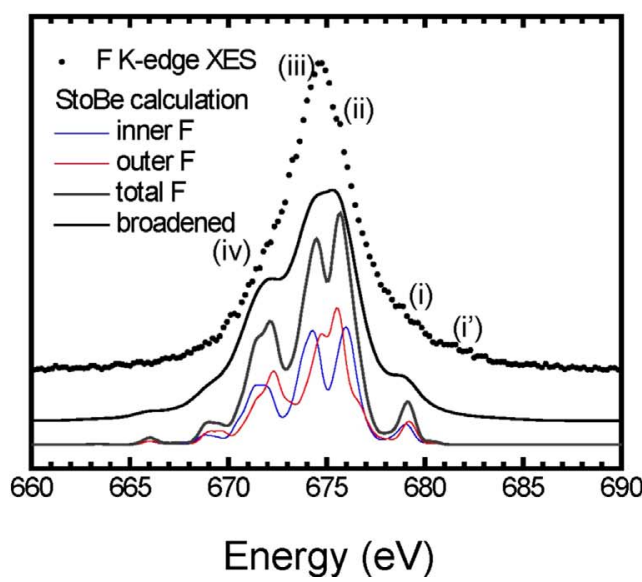

FIG. 6. (Color online) The (a) $\mathrm{N}$ and (b) F $K$-edge x-ray emission spectra of $\mathrm{F}_{16} \mathrm{CuPc}$. The corresponding StoBe calculated emission spectra (with site-specific contributions) are displayed beneath each experimental spectrum.

data. The difference is likely due to radiation damage to the samples in the earlier study, since those samples were not translated during measurement. ${ }^{4}$

The N $K$-edge XES spectra should reflect contributions from both the aza and inner nitrogen atoms. We have plotted the individual (chemical site-specific) and summed calculated contributions to the $\mathrm{N} K$-edge nonresonant XES beneath the experimental result in Fig. 6(a). We note excellent agreement in terms of the spectral shape between experiment and theory, with the theoretical calculation reproducing all peaks (i) $-(\mathrm{v})$. This allows us to identify feature (v) as being associated with transitions on the inner $\mathrm{N}$ atom involving the HOMO state. The relative intensity of this peak (compared to the other $K$-edge XES spectra) suggests a significant nitrogen contribution toward the $\mathrm{HOMO}$ state of $\mathrm{F}_{16} \mathrm{CuPc}$, consistent with the strong $\mathrm{C}-\mathrm{N}$ related $\pi-\pi^{*}$ shake-up feature observed in Fig. 2. Closer inspection reveals that this peak and the majority of the features apart from the main peak (i) are due mainly to contributions from the inner nitrogen, while the prominent main feature of the emission spectrum, peak (i) is derived largely from the aza mesobridging nitrogen atoms. The simulated XES spectra presented in Fig. 6 reflect the total, spherically averaged transitions, but similarly to the XAS we can consider the geometrical arrangement of the emitting molecule and the XES detector. For XES, we can then think of the spectra obtained as looking at the planar molecule "face-on" or "edge-on." Only in the latter "edgeon" orientation will the occupied $\pi$ states contribute to the XES. If we consider the $z$-axis as being perpendicular to the plane of the molecule then emission in a "face-on" orientation is essentially the projection of the occupied $2 p$ PDOS along the two orthogonal $x$ - and $y$-axes taking into account the transition matrix element involving the core $1 \mathrm{~s}$ electron on each atomic site. Thus the emission can be considered as an average over all possible emission polarization vectors perpendicular to the normal of the molecule (the $z$-axis) and is thus from occupied in-plane $\sigma$-like states only. By contrast, in the "edge-on" orientation the emission spectrum will be essentially the projected occupied $2 p$ PDOS along two orthogonal axes, the $z$-axis and an axis in the plane of the molecule (such as the $x$ - or $y$-axes) modified by the appropriate transition matrix element. The "edge-on" emission is then an average of all possible emission polarization vectors perpendicular to a given axis in the plane of the molecule, where we must average over directions along one of the isoindoline arms or between the isoindoline arms. Thus the "edge-on" emission will have contributions from both the occupied $\pi$ states and an average contribution of the in-plane occupied $\sigma$ states.

Note that for emission in the edge-on orientation that peaks (iv) and (v) are $\sim 60 \%$ and $\sim 15 \%$, respectively, of the peak intensity of feature (i), declining to $\sim 32 \%$ and $\sim 6 \%$ respectively in the face-on geometry. Our expectation from the XAS analysis which indicates an azimuthally averaged angle of the molecule of $\sim 45^{\circ}$ to the surface, is of a mixture of emission from the $2 p$ component of the states projected into the plane of the molecule and perpendicular to the plane of the molecule, but favoring the former. The geometry of the $\mathrm{x}$-ray emission measurements is, in all cases presented here, with the incident light at angle of $45^{\circ}$ to the surface and thus we expect a distribution of "edge-on" and "face-on" views of the molecule. In the case of the nonresonant XES spectra, the spherically averaged total transitions are appropriate to compare with the experimental spectra in this context.

Likewise, Fig. 6(b) presents the experimental F $K$-edge emission spectra and the theoretical chemical site-specific contributions to the XES from the fluorine atoms (both innermost and outermost atoms of the benzene ring bonded to $\mathrm{C}_{3}$ and $\mathrm{C}_{4}$, respectively). For the discussion here we have chosen first to calculate the F $K$-edge XES with a $0.8 \mathrm{eV}$ Gaussian FWHM to approximate both instrumental and corehole lifetime effects in a similar fashion to both the $\mathrm{C}$ and $\mathrm{N} K$ edges so as to allow easier comparison between molecular orbitals in the $\mathrm{C}$ and $\mathrm{N} K$-edge XES spectra. The final total F $K$-edge XES spectrum is further broadened by a 1.2 eV FWHM Lorentzian line shape. Three main spectral features are identified (i)-(iii), in agreement with the earlier report by Kurmaev et al. ${ }^{4}$ From comparison with the theoretical calculations, both PDOS and XES, we can attribute shoulder (i) in the experimental data as that representing transitions to the F $1 s$ core level from in-plane molecular orbitals in the same energy region as those contributing to the emission of $\mathrm{N}$ (i) feature with an almost negligible contribution arising from transitions from the HOMO which nevertheless has a finite projected density of states on both $\mathrm{F}$ 
sites. A very weak shoulder ( $\left.\mathrm{i}^{\prime}\right)$ is observed at higher photon energies (i.e., lower binding energies), which may be signature of transitions involving the HOMO, further supporting our assignment of peak (i). Feature (iii) is essentially emission from the in-plane $\mathrm{F} 2 p$ orbitals which form $\sigma$ bonds with their adjacent $C$ neighbors $\left(C_{3}\right.$ and $\left.C_{4}\right)$, while both features (ii) and (iv), have much greater prominence in the edge-on emission spectra (not shown) due to their majority out-of plane character and lie at a lower binding energy (ii) or a higher binding energy (iv) with the region in between completed by the almost exclusively in-plane F $2 p$ component. Similar to the N K edge, in comparison between the observed nonresonant XES spectrum and the calculated total, spherically averaged XES transitions, there is a slight favoring of emission from the in-plane component. Unlike the $\mathrm{N} K$-edge, all of the peaks/shoulders are equally derived from both fluorine environments (i.e., inner and outer). It is worth highlighting that the element-specific nature of XES, combined with density-of-states calculations where one can also calculate the PDOS and XES according to the geometry of the molecule, makes such conclusions easier to ascertain from theoretical computations than from traditional valenceband PES measurements of organic materials.

The interpretation of emission spectra in the presence of more than one core hole with a wide range of binding energies (chemical shifts) on differing atomic sites is complicated due to the presence of simultaneous fluorescent decay channels at sufficiently high excitation energies. As a result the $\mathrm{C} K$-edge emission spectrum of $\mathrm{F}_{16} \mathrm{CuPc}$ is expected to show several additional features, or more complex behavior, when compared to $\mathrm{CuPc}$. Figure 7 shows both the translated and stationary $\mathrm{C} K$-edge XES of (a) $\mathrm{F}_{16} \mathrm{CuPc}$ and (b) CuPc. We note agreement with the $\mathrm{C} K$-edge XES spectra of $\mathrm{F}_{16} \mathrm{CuPc}$ and $\mathrm{CuPc}$ of our stationary samples and the results by Kurmaev et $a l^{4}{ }^{4}$ For these cases, only a broadened emission spectrum was noted between fluorinated and nonfluorinated associated with the spreading of the $\mathrm{C} 1 s$ core level. This is in stark contrast to the dramatic difference observed between translated (nonbeam-damaged) $\mathrm{F}_{16} \mathrm{CuPc}$ and $\mathrm{CuPc}$ emission spectra. Most notably, additional sharp features are clearly observed in $\mathrm{F}_{16} \mathrm{CuPc}$ which are absent for $\mathrm{CuPc}$. One can consider the above threshold $\mathrm{C} K$-edge XES spectrum from $\mathrm{CuPc}$ to be the sum of pyrrole and phenyl $\mathrm{C} 2 p \rightarrow \mathrm{C} 1 s$ transitions. For $\mathrm{F}_{16} \mathrm{CuPc}$, the $\mathrm{C} K$-edge spectrum is instead the sum of three distinct sets of $\mathrm{C} 2 p \rightarrow \mathrm{C} 1 s$ transitions where the phenyl (i.e., $\mathrm{C}-\mathrm{C}, \mathrm{C}-\mathrm{H}$ ) contribution is split into fluorine (inner and outer) phenyl carbons $\left(\mathrm{C}-\mathrm{F}, \mathrm{C}_{3,4}\right)$ and link phenyl carbons $\left(\mathrm{C}-\mathrm{C}, \mathrm{C}_{2}\right)$, consistent with the $\mathrm{C} 1 s$ XPS. The simulated $\mathrm{C} K$-edge XES spectra in Fig. 7 are the equally weighted combined sum of the total spherically averaged dipole transition moments. If we consider the $\mathrm{CuPc}$ case first, excellent agreement is observed between the spectrum from the translated sample and the calculation for all features. Significantly, transitions involving HOMO to the pyrrole and phenyl atoms can be distinguished as the peaks at photon energies $h \nu=283.7 \mathrm{eV}$ (i) and $282.5 \mathrm{eV}$ (ii), respectively. Likewise, there is excellent agreement between theory and experiment for the case of $\mathrm{F}_{16} \mathrm{CuPc}$. For this case the $\mathrm{HOMO}$ is more difficult to observe than for the $\mathrm{CuPc}$. This can be understood in terms of the C-F contribution. The C-6F $\left(\mathrm{C}_{3,4}\right)$
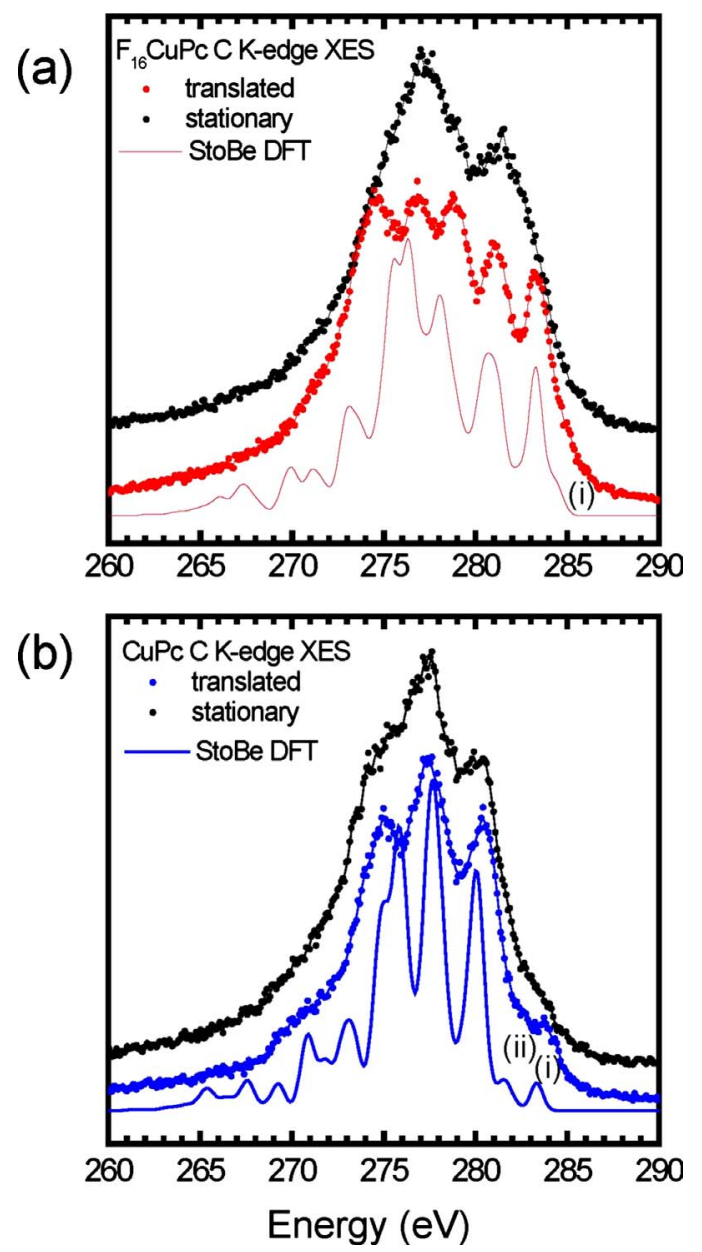

FIG. 7. (Color online) (a) $\mathrm{C} K$-edge XES from a stationary $\mathrm{F}_{16} \mathrm{CuPc}$ film (black dots) and a translated $\mathrm{F}_{16} \mathrm{CuPc}$ film (red dots). Also displayed is the StoBe calculated nonresonant emission spectrum (red line). (b) $\mathrm{C} K$-edge XES from a stationary $\mathrm{CuPc}$ film (black dots) and a translated $\mathrm{CuPc}$ film (blue dots), with a calculated nonresonant emission spectrum (blue line). A 3 point-binomial smoothing has been used (thin lines) for each experimental spectrum to guide the eye.

atoms contribute strongly to the HOMO- 1 region $\left(b_{2 \mathrm{~g}}, b_{1 \mathrm{u}}\right.$, and $e_{\mathrm{g}}$ MOs) and weakly to the $\operatorname{HOMO}\left(a_{1 \mathrm{u}}\right)$. Due to the core-level shifts between these carbons within the benzene rings $\left(\mathrm{C}-\mathrm{F}, \mathrm{C}_{3,4}\right)$ and the pyrrole carbons $\left(\mathrm{C}-\mathrm{N}, \mathrm{C}_{1}\right)$ observed in the $\mathrm{C} 1 s$ XPS, the peak associated with the HOMO-1 $\rightarrow\left(\mathrm{C}-\mathrm{F}, \mathrm{C}_{3,4}\right) \mathrm{C} 1 \mathrm{~s}$ transitions dominate the high photon energy side of the emission spectrum. However, a weak high energy shoulder (i) can be identified which is considered to reflect overlapping transitions involving the HOMO associated with the outermost fluorinated $\mathrm{C}$ atoms $\left(\mathrm{C}_{4}\right)$ according to our calculations.

\section{CARBON $K$-EDGE RESONANT X-RAY EMISSION}

By using incident energies close to the core absorption edge, it is possible to glean additional site-specific information using RXES. We have employed $\mathrm{C} K$-edge RXES to distinguish contributions to the carbon-related electronic structure of $\mathrm{F}_{16} \mathrm{CuPc}$. This approach has been successfully 


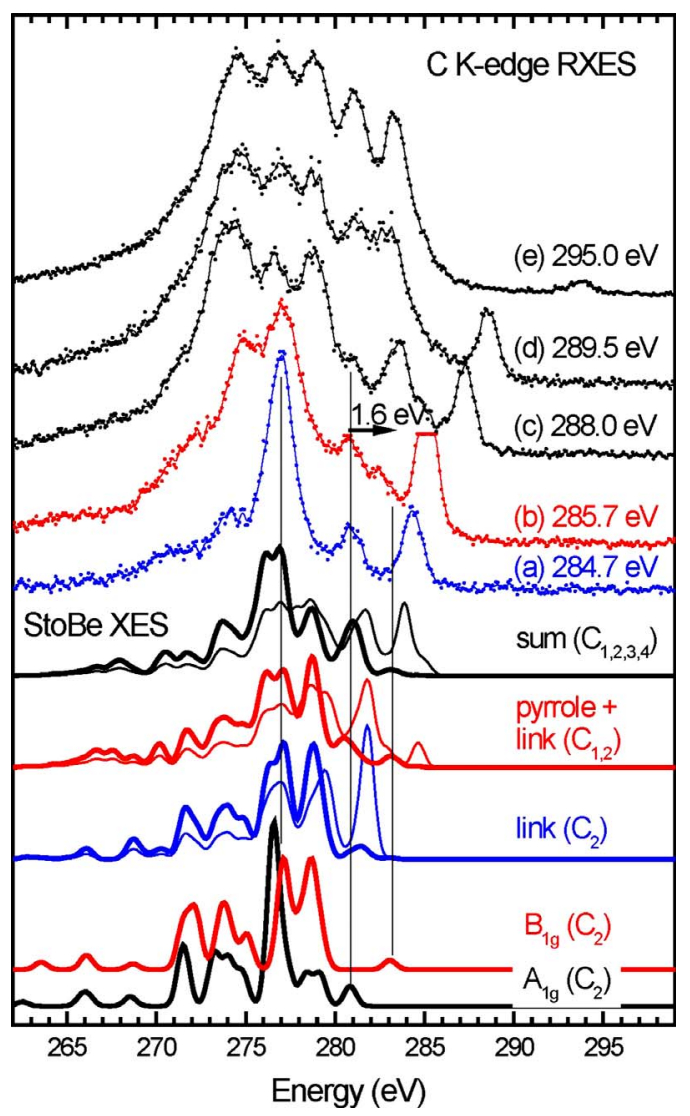

FIG. 8. (Color online) The experimental C $K$-edge RXES spectra $\mathrm{F}_{16} \mathrm{CuPc}$ (a)-(e) as a function of excitation energy (filled circles). The elastic peak lies at the incident energy used in each case and for (b) $h \nu=285.7 \mathrm{eV}$ the intensity of the elastic peak has been truncated. Also shown underneath the experimental spectra are the StoBe simulated emission spectra corresponding to contributions from, in ascending order, solely those transitions arising from $a_{1 \mathrm{~g}}$ symmetry MOs on the link $\mathrm{C}_{2}$ site (C-C bond) in face-on geometry; then the spectrum arising solely from $b_{1 \mathrm{~g}}$ symmetry MOs on the $\mathrm{C}_{2}$ sit in face-on geometry; then the total spectrum arising from the $\mathrm{C}_{2}$ carbon site (blue line) shown for both face-on emission geometry (thick line) and edge-on emission geometry (thin line); then the sum of the spectra arising from $\mathrm{C}_{1}(\mathrm{C}-\mathrm{N})$ and $\mathrm{C}_{2}(\mathrm{C}-\mathrm{C})$ link and pyrrole) carbon sites (red line) again in both face-on and edge-on geometries; and finally the sum of all (sum) carbon sites (black line) in both face-on and edge-on geometries.

employed recently to investigate the electronic structure of the organic molecule $\mathrm{Alq}_{3} \cdot{ }^{19}$ Figure 8 displays the $\mathrm{C} K$-edge RXES spectra for $\mathrm{F}_{16} \mathrm{CuPc}$, the excitation energies corresponding to highlighted peaks of the corresponding $\mathrm{C} K$-edge XAS in Fig. 5(b). We note large spectral changes in the emission spectra as the incident photon energy is increased from threshold at $284.7-288 \mathrm{eV}$ in the $\mathrm{C} K$-edge RXES of $\mathrm{F}_{16} \mathrm{CuPc}$. Subsequently, the spectra tend toward the above threshold spectrum $\left(h \nu_{\text {excite }}=295 \mathrm{eV}\right)$. This is understood in terms of RXES spectra (a)-(c) lying below the ionization thresholds of any of the $\mathrm{C}$ sites, where each spectrum, reflects the emission accompanying the excitation of an electron from a $1 s$ core level into $\pi^{*}$ states on that site where differing sites contribute according to the excitation energy. In order to interpret the RXES spectra displayed in Fig. 8 it is therefore necessary to refer to assignment of the spectral peaks in the C $K$-edge XAS spectrum already discussed in Sec. V. Note also that both symmetry selectivity and spatial selectivity play significant roles in determining the states contributing to the RXES spectra. Spatial selectivity in that for a $90^{\circ}$ scattering geometry the excitation into a $\pi^{*}$ molecular orbital perfectly aligned with the linearly polarized light will result in the observed de-excitation or emission from the occupied $\sigma$ orbitals only. ${ }^{56}$ Thus the threshold $\mathrm{C} K$-edge RXES spectra exciting into the $\pi^{*}$ LUMO will reflect the "face-on" view of the projected PDOS and RXES arising from the occupied $\sigma$ orbitals. Both the "face-on" and "edge-on" XES spectra are presented in Fig. 8 and will be discussed below. Symmetry selectivity plays a role in that for a centrosymmetric molecule such as $\mathrm{F}_{16} \mathrm{CuPc}$, the RXES must first obey conservation of parity such that excitations to gerade molecular orbitals, such as the Pc-ligand-derived $e_{\mathrm{g}}$ LUMO, must be accompanied by RXES from occupied states also of gerade parity, such as the $a_{1 \mathrm{~g}}$ and $b_{1 \mathrm{~g}}$ occupied molecular orbitals as will be discussed below. Such symmetry selective selection rules are discussed by Luo et al. ${ }^{56,57}$

Figure 8 plots both the experimental C $K$-edge RXES of $\mathrm{F}_{16} \mathrm{CuPc}$ and the corresponding StoBe calculated XES spectra associated with transitions involving the (a) link carbon, (b) link and pyrrole, and (c) all carbon environments with each calculated spectrum presented as both face-on and edge-on spectra summing over all occupied molecular-orbital symmetries. Nearly all features (excluding the elastic peak), both in terms of relative intensities and energetic spacing, are reproduced for (a)-(c). These nonsymmetry resolved calculations are able to best reproduce spectra (b) and (c), most notably the emergence of the higher photon energy spectral features (at $\sim 280-284 \mathrm{eV}$ ) relating to the fluorescent decay transitions: HOMO, HOMO- $1 \rightarrow \mathrm{C}-\mathrm{F}\left(\mathrm{C}_{3,4}\right) \mathrm{C} 1 \mathrm{~s}$ as the incident energy is increased from 285.7 to $288 \mathrm{eV}$.

We can consider the RXES spectrum (a) to represent transitions solely involving the link phenyl carbon sites $\left(\mathrm{C}_{2}\right)$, first as their $1 s$ binding energy is lowest, and the onset of the calculated $\mathrm{C} K$-edge XAS including all of peak a in Fig. 5(a) is due to transitions to the $\pi^{*} e_{\mathrm{g}}$ LUMO on this C site only. It should be noted that there is calculated to be a very small intensity $\sigma^{*}$-like component of the XAS at $\sim 284$ and $285 \mathrm{eV}$ comprising transitions to the $\mathrm{Cu} 3 d_{x^{2}-y^{2}}$ derived $a_{1 \mathrm{u}}$ orbital from the link and pyrrole sites respectively. The energetic positioning of these transitions are likely to be too low, as we have commented in the case of the $\mathrm{N} K$-edge XAS spectrum, perhaps by at least $\sim 1 \mathrm{eV}$. For a $D_{4 h}$ molecule, the symmetry selection rules of Luo et al. indicate that only those XES transitions derived from the occupied molecular orbitals of $a_{1 \mathrm{~g}}, a_{2 \mathrm{~g}}, b_{1 \mathrm{~g}}$, and $b_{2 \mathrm{~g}}$ will participate in the RXES when the excitation is resonant with the unoccupied $e_{\mathrm{g}}$ orbital. At the bottom of Fig. 8 is the calculated $1 s-2 p$ RXES spectrum arising solely from the $a_{1 \mathrm{~g}}$ symmetry occupied molecularorbital components located on the link $\left(\mathrm{C}_{2}\right)$ site. For comparison we also show the $b_{1 \mathrm{~g}}$ component part of the RXES on the same $\mathrm{C}_{2}$ site, particularly as the Pc-derived HOMO is of $b_{1 \mathrm{~g}}$ symmetry. For spectra (a) and (b) no out-of-plane $\pi$-like molecular orbital contributes to the emission due to the aforementioned $1 s \rightarrow \pi^{*} ; \sigma \rightarrow 1 s$ spatial selectivity as a result of the positioning of the XES spectrometer in a $90^{\circ}$ 
scattering geometry. This is clearly evident from comparison of the calculated XES emission in face-on and edges-on geometries with the experimental spectra, most notably 280$282 \mathrm{eV}$. In examining spectrum (a) and the experimentally observed very prominent peak (at $\sim 277 \mathrm{eV}$ ), we see a much closer correspondence between the emission from the inplane $a_{1 \mathrm{~g}}$ symmetry molecular orbitals than for any other combination of molecular orbitals (not all shown), whether the sum of all $a_{1 \mathrm{~g}}, a_{2 \mathrm{~g}}, b_{1 \mathrm{~g}}$, and $b_{2 \mathrm{~g}}$, or even the sum of all the in-plane components (gerade and ungerade) which is shown as the calculated face-on (blue) spectrum in Fig. 8. While there is a possibility of transitions on the $\mathrm{C}_{2}$ site to the small site-projected component of the $a_{1 \mathrm{u}} \mathrm{Cu} 3 d$-derived LUMO, of the anticipated emission from ungerade symmetries, only the $b_{2 \mathrm{u}}$ has any prominent $\sigma$-like component coincident with the peak at $\sim 277 \mathrm{eV}$ but this is not expected to contribute. All other ungerade, and especially those occupied orbitals whether gerade or ungerade of $\pi$ symmetry, have much more spectral weight in the region from $277-282 \mathrm{eV}$, and can be seen not to contribute to the threshold spectrum (a). In spectrum (b), the excitation energy is now resonant with the Pcderived $e_{\mathrm{g}}$ LUMO on the pyrrole $\mathrm{C}$ site $\left(\mathrm{C}_{1}\right)$ while also exciting transitions on the link $\mathrm{C}_{2}$ site to higher $\pi^{*}$ unoccupied orbitals on that site. Thus the observed emission spectrum is no longer restricted to a specific set of molecular-orbital symmetries on a single atomic site but becomes a more complex superposition of these spectra according to the absorption transition strength on each $\mathrm{C}$ site, the spatial orientation and symmetries of the selected unoccupied orbitals on these sites (in this case just $\mathrm{C}_{1}$ and $\mathrm{C}_{2}$ ), and hence the weighting and selected symmetries and orientations of the molecular orbitals on each site that contribute to the observed emission. We do not attempt to model this in detail, but instead draw attention to the occupied electronic structure near the Fermi level which is no longer obscured by the overlapping wings of the elastic peak in spectrum (b) as it was in spectrum (a). The vertical lines drawn in Fig. 8 indicate the positions of the XES transitions from the Pc-derived HOMO $b_{1 \mathrm{~g}}$ level as located on the $\mathrm{C}_{2}$ site; the most intense feature of spectrum (a) which appears in close coincidence with most prominent feature of the emission from the $a_{1 \mathrm{~g}}$ symmetry orbitals in emission from the $\mathrm{C}_{2}$ site and the highest transition energy associated with emission from the $a_{1 \mathrm{~g}}$ HOMO orbitals on the $\mathrm{C}_{2}$ sites. The latter feature appears to be reproduced in both spectrum (a) and in spectrum (b) but displaced to an emission energy which is $1.6 \mathrm{eV}$ higher, in sharp contrast to the observed chemical shift in the ionization energies between $\mathrm{C}_{1}$ and $\mathrm{C}_{2}$ which is only $\sim 1.4 \mathrm{eV}$. Although the $b_{1 \mathrm{~g}}$ on $\mathrm{C}_{2}$ is highlighted and compared to an observed feature in spectrum (b), it is unclear whether this HOMO level can be observed independently of transitions from any other molecular orbital.

As one proceeds from spectrum (c) upwards toward higher excitation energies, two effects come into play, the first being that we begin to excite the $\mathrm{C} 1 \mathrm{~s} \rightarrow \pi^{*}$ transitions on the fluorinated $\mathrm{C}$ sites $\left(\mathrm{C}_{3}, \mathrm{C}_{4}\right)$. This then results in a switching on of the face-on emission arising from the inplane $\sigma$ orbitals on these $\mathrm{C}$ sites. Almost simultaneously with these higher excitation energies is the progressive transition from the $\pi^{*}$ region in the XAS of $\mathrm{C}_{2}$ (and subsequently $\mathrm{C}_{1}$ ) into the $\sigma^{*}$ region [see Fig. 5(a)], which allows emission from the occupied $\pi$-like molecular orbitals, principally the $e_{\mathrm{g}}$, but also $\pi$-like $a_{1 \mathrm{u}}, a_{2 \mathrm{u}}, b_{1 \mathrm{u}}$ and $b_{2 \mathrm{u}}$ symmetry orbitals which tend to comprise the majority of the HOMO states nearest the Fermi level. Their contributions can be estimated from the calculated edge-on XES spectra shown for the link $\mathrm{C}_{2}$, the link and the pyrrole $\mathrm{C}_{1}$ and the sum of these and from the remaining $C$ sites $\left(C_{3}\right.$ and $\left.C_{4}\right)$ shown in Fig. 8. Thus the additional peaks that are to observed gaining further prominence in spectra (d) and (e) arise due to emission from these occupied $\pi$ HOMO states. As the $\sigma^{*}$ component increases toward higher photon energy so the emission from the $\pi$ states plays a greater role in the observed emission spectra (only to be seen in edge-on emission) while the while the contribution of the occupied $\sigma$ states decreases. Once sufficiently above threshold the spherically averaged X-ray emission is a good approximation of the observed nonresonant XES spectra as previously indicated in Fig. 7

\section{CONCLUSION}

The element-specific bulk electronic structure of pristine copper hexadecafluorophthalocyanine films has been investigated using synchrotron-radiation excited XAS and XES at the $\mathrm{C}, \mathrm{N}$, and $\mathrm{F} K$ edges. In contrast to previous reports, we find the $\mathrm{C} K$-edge XES of $\mathrm{F}_{16} \mathrm{CuPc}$ to be distinctly different to $\mathrm{CuPc}$ when the effects of $\mathrm{X}$-ray induced damage are minimized. High resolution XES of the $\mathrm{F}_{16} \mathrm{CuPc}$ films were directly compared with ground-state DFT (within the StoBe framework) of the occupied element-specific $p$-like molecular orbitals. Excellent agreement between theory and experiment of all three light element $\mathrm{K}$ edges, allowed us to confidently assign spectral features in terms of transitions with core levels involving specific molecular orbitals. Furthermore, $\mathrm{C} \mathrm{K}$-edge RXES of $\mathrm{F}_{16} \mathrm{CuPc}$ displays an evolution of spectral features with increasing photon energy, which can be understood in terms of increasing contributions from the various $\mathrm{C}$ atom electronic environments, symmetries and molecular orbitals. This work provides an improved detailed knowledge of the ground-state electronic structure of this technologically important organic molecule necessary to development our understanding of organic interface formation. This study also highlights the benefits of XES (and RXES) over traditional photoemission for studying the occupied molecular orbitals of organic molecules.

\section{ACKNOWLEDGMENTS}

This work was supported in part by the NSF under Grant No. CHE-0807368, and by the U.S. Air Force Office of Scientific Research under Grant No. FA9550-06-1-0157. This material is based upon works supported by the Science Foundation Ireland under Grant No. 04/BR/P0254. C. McGuinness would also like to thank L.G.M. Pettersson, F. Hermann, and B. Brena for helpful discussions. The NSLS is supported by the U.S. Department of Energy, Office of Science, Office of Basic Energy Sciences, under Contract No. DE-AC02-98CH10886 
*Present address: Department of Chemistry, University of Nevada Las Vegas, 4505 Maryland Parkway, Las Vegas, NV 89154.

${ }^{\dagger}$ ksmith@bu.edu

${ }^{1}$ E. Tosatti, M. Fabrizio, J. Tobik, and G. E. Santoro, Phys. Rev. Lett. 93, 117002 (2004).

${ }^{2}$ S. Heutz et al., Adv. Mater. 19, 3618 (2007).

${ }^{3}$ A. Bohler, P. Urbach, D. Ammermann, and W. Kowalsky, Mater. Sci. Eng., B 51, 58 (1998).

${ }^{4}$ E. Z. Kurmaev et al., Phys. Rev. B 64, 045211 (2001).

${ }^{5}$ G. Giovannetti, G. Brocks, and J. van den Brink, Phys. Rev. B 77, 035133 (2008).

${ }^{6}$ Z. Bao, A. J. Lovinger, and A. Dodabalapur, Appl. Phys. Lett. 69, 3066 (1996).

${ }^{7}$ Z. A. Bao, A. J. Lovinger, and J. Brown, J. Am. Chem. Soc. 120, 207 (1998)

${ }^{8}$ J. Wang, H. B. Wang, X. J. Yan, H. C. Huang, D. Jin, J. W. Shi, Y. H. Tang, and D. H. Yan, Adv. Funct. Mater. 16, 824 (2006).

${ }^{9}$ Q. X. Tang, H. X. Li, Y. L. Liu, and W. P. Hu, J. Am. Chem. Soc. 128, 14634 (2006).

${ }^{10}$ H. Peisert, M. Knupfer, T. Schwieger, G. G. Fuentes, D. Olligs, J. Fink, and T. Schmidt, J. Appl. Phys. 93, 9683 (2003).

${ }^{11}$ K. M. Lau, J. X. Tang, H. Y. Sun, C. S. Lee, S. T. Lee, and D. Yan, Appl. Phys. Lett. 88, 173513 (2006).

${ }^{12}$ O. V. Molodtsova, M. Grobosch, M. Knupfer, and V. Y. Aristov, Appl. Phys. Lett. 91, 244103 (2007).

${ }^{13}$ W. Chen, S. Chen, H. Huang, D. C. Qi, X. Y. Gao, and A. T. S. Wee, Appl. Phys. Lett. 92, 063308 (2008).

${ }^{14}$ H. Vazquez, Y. J. Dappe, J. Ortega, and F. Flores, J. Chem. Phys. 126, 144703 (2007).

${ }^{15}$ M. G. Betti, A. Kanjilal, C. Mariani, H. Vazquez, Y. J. Dappe, J. Ortega, and F. Flores, Phys. Rev. Lett. 100, 027601 (2008).

${ }^{16}$ D. R. T. Zahn, G. N. Gavrila, and M. Gorgoi, Chem. Phys. 325, 99 (2006).

${ }^{17}$ L. L. Chua, J. Zaumseil, J. F. Chang, E. C. W. Ou, P. K. H. Ho, H. Sirringhaus, and R. H. Friend, Nature (London) 434, 194 (2005).

${ }^{18}$ Y. Zhang, T. Learmonth, S. Wang, A. Y. Matsuura, J. Downes, L. Plucinski, S. Bernardis, C. O'Donnell, and K. E. Smith, J. Mater. Chem. 17, 1276 (2007).

${ }^{19}$ A. DeMasi et al., J. Chem. Phys. 129, 224705 (2008).

${ }^{20}$ J. E. Downes, C. McGuinness, P.-A. Glans, T. Learmonth, D. Fu, P. Sheridan, and K. E. Smith, Chem. Phys. Lett. 390, 203 (2004).

${ }^{21}$ Y. Zhang, S. Wang, T. Learmonth, L. Plucinski, A. Y. Matsuura, S. Bernardis, C. O'Donnell, J. E. Downes, and K. E. Smith, Chem. Phys. Lett. 413, 95 (2005).

${ }^{22}$ Y. Zhang, S. Wang, A. Demasi, I. Reid, L. F. J. Piper, A. Y. Matsuura, J. E. Downes, and K. E. Smith, J. Mater. Chem. 18, 1792 (2008).

${ }^{23}$ B. N. Holland, N. Peltekis, T. Farrelly, R. G. Wilks, G. Gavrila, D. R. T. Zahn, C. McGuinness, and I. T. McGovern, Phys. Status Solidi B 246, 1546 (2009).

${ }^{24}$ V. Y. Aristov, O. V. Molodtsova, V. Maslyuk, D. V. Vyalikh, V. M. Zhilin, Y. A. Ossipyan, T. Bredow, I. Mertig, and M. Knupfer, Appl. Surf. Sci. 254, 20 (2007).

${ }^{25}$ F. Evangelista, V. Carravetta, G. Stefani, B. Jansik, M. Alagia, S. Stranges, and A. Ruocco, J. Chem. Phys. 126, 124709 (2007).

${ }^{26}$ T. Ikame, K. Kanai, Y. Ouchi, E. Ito, A. Fujimori, and K. Seki, Chem. Phys. Lett. 413, 373 (2005).
${ }^{27}$ K. K. Okudaira, H. Setoyama, H. Yagi, K. Mase, S. Kera, A. Kahn, and N. Ueno, J. Electron Spectrosc. Relat. Phenom. 137. 140, 137 (2004).

${ }^{28}$ K. Hermann, L. G. M. Pettersson, M. E. Casida, C. Daul, A. Goursot, A. Koester, E. Proynov, A. St-Amant, and D. R. Salahub, StoBe-deMon version 3.0, 2008.

${ }^{29}$ P. Skytt, J. Guo, N. Wassdahl, J. Nordgren, Y. Luo, and H. Agren, Phys. Rev. A 52, 3572 (1995).

${ }^{30}$ X. Chen, P.-A. Glans, X. Qiu, S. Dayal, W. D. Jennings, K. E. Smith, C. Burda, and J. Guo, J. Electron Spectrosc. Relat. Phenom. 162, 67 (2008).

${ }^{31}$ J. Nordgren, G. Bray, S. Cramm, R. Nyholm, J. E. Rubensson, and N. Wassdahl, Rev. Sci. Instrum. 60, 1690 (1989).

${ }^{32}$ X-ray Data Booklet, edited by A. C. Thompson, David Attwood, Eric Gullikson, Malcolm Howells, Kwang-Je Kim, Janos Kirz, Jeffery Kortright, Ingolf Lindau, Piero Pianetta, Arthur Robinson, James Scofield, James Underwood, Douglas Vaughen, Gwyn Williams, and Herman Winick (Lawrence Berkeley National Laboratory, University of California, Berkeley, CA, 2001).

${ }^{33}$ H. Ogasawara, B. Brena, D. Nordlund, M. Nyberg, A. Pelmenschikov, L. G. M. Pettersson, and A. Nilsson, Phys. Rev. Lett. 89, 276102 (2002).

${ }^{34}$ J. P. Perdew and Y. Wang, Phys. Rev. B 33, 8800 (1986).

${ }^{35}$ A. D. Becke, Phys. Rev. A 38, 3098 (1988).

${ }^{36}$ A. Fohlisch, J. Hasselstrom, P. Bennich, N. Wassdahl, O. Karis, A. Nilsson, L. Triguero, M. Nyberg, and L. G. M. Pettersson, Phys. Rev. B 61, 16229 (2000).

${ }^{37}$ C. Kolczewski et al., J. Chem. Phys. 115, 6426 (2001).

${ }^{38}$ O. V. Molodtsova, M. Knupfer, V. V. Maslyuk, D. V. Vyalikh, V. M. Zhilin, Y. A. Ossipyan, T. Bredow, I. Mertig, and V. Y. Aristov, J. Chem. Phys. 129, 154705 (2008).

${ }^{39}$ W. Kutzelnigg, U. Fleischer, and M. Schindler, NMR Basic Principles and Progress (Springer, Heidelberg, 1990).

${ }^{40}$ S. Huzinaga, J. Chem. Phys. 42, 1293 (1965).

${ }^{41}$ T. Schwieger, H. Peisert, M. S. Golden, M. Knupfer, and J. Fink, Phys. Rev. B 66, 155207 (2002).

${ }^{42}$ L. Ottaviano, S. DiNardo, L. Lozzi, M. Passacantando, P. Picozzi, and S. Santucci, Surf. Sci. 373, 318 (1997).

${ }^{43}$ H. Peisert, M. Knupfer, and J. Fink, Surf. Sci. 515, 491 (2002).

${ }^{44}$ B. Brena et al., Phys. Rev. B 70, 195214 (2004).

${ }^{45}$ I. Biswas, H. Peisert, M. Nagel, M. B. Casu, S. Schuppler, P. Nagel, E. Pellegrin, and T. Chasse, J. Chem. Phys. 126, 174704 (2007).

${ }^{46}$ L. Zhang, H. Peisert, I. Biswas, M. Knupfer, D. Batchelor, and T. Chasse, Surf. Sci. 596, 98 (2005).

${ }^{47}$ A. Cossaro, D. Cvetko, G. Bavdek, L. Floreano, R. Gotter, A. Morgante, F. Evangelista, and A. Ruocco, J. Phys. Chem. B 108, 14671 (2004).

${ }^{48}$ L. Ottaviano, L. Lozzi, F. Ramondo, P. Picozzi, and S. Santucci, J. Electron Spectrosc. Relat. Phenom. 105, 145 (1999).

${ }^{49}$ S. Kera, M. B. Casu, A. Scholl, T. Schmidt, D. Batchelor, E. Ruhl, and E. Umbach, J. Chem. Phys. 125, 014705 (2006).

${ }^{50}$ J. Stöhr, NEXAFS Spectroscopy (Springer, Berlin, 1992).

${ }^{51}$ V. Y. Aristov, O. V. Molodtsova, V. V. Maslyuk, D. V. Vyalikh, V. M. Zhilin, Y. A. Ossipyan, T. Bredow, I. Mertig, and M. Knupfer, J. Chem. Phys. 128, 034703 (2008).

${ }^{52}$ D. J. Bristow and G. M. Bancroft, J. Am. Chem. Soc. 105, 5634 (1983).

${ }^{53}$ M. M. Brzhezinskaya, V. E. Muradyan, N. A. Vinogradov, A. B. 
Preobrajenski, W. Gudat, and A. S. Vinogradov, Phys. Rev. B 79, 155439 (2009).

${ }^{54}$ J. Nordgren and N. Wassdahl, J. Electron Spectrosc. Relat. Phenom. 72, 273 (1995).

${ }^{55}$ J. Nordgren, P. Glans, K. Gunnelin, J. Guo, P. Skytt, C. Sathe, and N. Wassdahl, Appl. Phys. A: Mater. Sci. Process. 65, 97 (1997).

${ }^{56}$ F. Gel'mukhanov and H. Agren, Phys. Rep. 312, 87 (1999).

${ }^{57}$ Y. Luo, H. Agren, and F. Gel'mukhanov, J. Phys. B 27, 4169 (1994). 